\title{
Emotion understanding of Turkish preschoolers and maternal emotional socialization ${ }^{12}$
}

\author{
Şükran K1lıç3 \\ Abide Güngör Aytar ${ }^{4}$
}

\begin{abstract}
The aim of the study is to investigate the relationship between emotion understanding and maternal emotional socialization responses to children's negative emotions. Emotion understanding was also investigated according to children's age and gender. The participants included 210 Turkish children and their mothers living in Ankara, Turkey. All children were recruited from kindergartens and all of them were between 48-72 month-olds. To gather data, Affect Knowledge Test (AKT) and Coping with Children's Negative Emotions Scale were used. Confirmatory Factor Analysis was performed for construct validity for AKT. Pearson correlation coefficients, ANOVA and posthoc tests were conducted. In this study, no relations were established between emotion understanding and the maternal emotional responses to children's negative emotions. It also has been revealed that 72 month-olds had better emotion understanding skills and emotion understanding did not change according to children's gender. It may be useful to include other important predictors of children's social and emotional competence and paternal responses for future studies.
\end{abstract}

Keywords: Emotional Understanding; Maternal Emotion Socialization; Turkish Preschoolers.

\section{Introduction}

Emotion understanding is one of the important components of the emotional competence for preschoolers (Denham, Mitchell-Copeland, Strandberg, Auerbach, \& Blair 1997). In addition, it is a significant task for preschoolers according to developmental perspective (Dunn, Bretherton, \& Munn, 1987). Emotion understanding which forms up a successful social interaction during childhood is generally defined as the cognitive representation of feelings and emotional behaviours of an individual (Meerum, Tergowt, \& Olthof, 1989, Gomez-Guerrero, 2003). Emotion understanding is defined as the ability to understand verbal and nonverbal expressions of one's emotion and others' emotions. More specifically, it has been suggested that it is a composite score which depends on multifaceted abilities, the ability to recognize other's emotional expression, the ability to perceive situational cues, the ability to consider other's emotional perspectives based on expressional and contextual clues (Denham, Blair, DeMulder, Levitas, Sawyer, \& Auerbach-Major,

\footnotetext{
1 This study was presented in 8th World Conference on Educational Sciences, Spain, Madrid.

2 This paper was produced from the first authors' doctoral dissertation title with The Relationship Between Children's Emotion Understanding Aged Between 48-72 Month-olds And Maternal Emotional Socialization Behavior

3 Assistant, Prof., Aksaray University, Faculty of Education, Department of Early Childhood and Education, kilic.sukran@gmail.com

${ }^{4}$ Prof. Dr., Gazi University, Faculty of Vocational Education, Department of Child Development and Education, abidegungor@gmail.com
} 
Kılıç, Ş., \& Güngör Aytar, A. (2016). Emotion understanding of Turkish preschoolers and maternal emotional socialization. International Journal of Human Sciences, 13(1), 2102-2112. doi:10.14687/ijhs.v13i1.3708

2003). During preschool period, children start developing skills which help them understand emotions as a part of a comprehensive emotional and cognitive development (Harris, 1989). Studies concerning emotion understanding report that preschoolers can recognize and label basic emotional facial expressions (Cutting \& Dunn, 1999). Furthermore preschoolers have affective perspective taking abilities (Denham, 1986). Already developed emotional vocabularies and social experiences of children enable them to understand the others' perspectives as well as to refer to emotions (Smith \& Walden, 1998). Numerous studies on emotion understanding of children assume that the most fundamental changes are detected between 36-72 month-olds (Dunn \& Hughes, 1998). Research conducted in the last two decades on emotional and cognitive development demonstrate that there is a developmental change in the emotion recognition abilities of children from 12 to 36 month-olds (Pons \& Harris, 2005). Whenever a drawing, photograph or picture showing an expression is showed to children in 48 and 60 month-olds, it is known that they can recognize happy, sad and angry facial expressions and label them correctly, as well (Camras \& Allison,1985). As children grow up, emotional facial expressions based clues are diminished and situational based clues are heightened. Morever their emotion situation knowledge and affective perspective skill continue to develop according to different studies (e.g., Gnepp, 1983).

Preschool period is a critical period for social and emotional competencies (Bronson, 2000) and in this period although children make friends, they still have close relations with their mothers (Denham, 1998). Mothers, one of the most significant adults enabling the socialization of children shape the emotional competence of their children with their responses against the emotions of their children (Eisenberg, Cumberland, \& Spinrad, 1998).

When taking the emotional socialization of children into consideration, specific behaviors of parents directly towards children are defined as "Emotion Socialization Responses" (Eisenberg et al., 1998). According to Gottman, Katz, \& Hooven (1996) socialization responses of parents include the adaptive and maladaptive thoughts, emotions and approaches that parents make in order to trigger childrens' emotional understanding. Denham (1998) states that there are three processes contributing to the explanation how social learning mechanisms are useful for emotional socialization: modeling, coaching and contingency. It is reported that supportive responses, positive emotional statements and experiences are the most useful implementations for socializing children (Denham, Bassett, \& Wyatt, 2010). Supportive responses of parents for child emotions (Eisenberg, Fabes, \& Murphy, 1996) ease the child to differentiate the children to differ their emotions (Fabes, Poulin, Eisenberg, \& Madden-Derdich, 2002). Fabes, Leonard, Kupanoff, \& Martin (2001) claim that there are six kinds of parental responses to struggle with children's negative emotions. These are problem-focused responses, emotion-focused responses, expressive encouragement, punitive reactions, minimization reactions, as well as parental distress reactions. Supportive responses include supports of mothers for coaching and teaching. Problem-focused responses are those which are most frequently used by parents for struggling with children's negative emotions. Emotion-focused responses reflect the parental responses to relax and calm child due to the trouble that the child is exposed. Expressive Encouragement reflects actively encouraging children's expression of negative emotions. Supportive responses make children more ready and sensitive to emotion learning compared to those responses of nonsupportive parental responses (Denham \& Grout, 1993). Coaching and contingent responses of parents such as problem solution or relaxation will produce more information on emotion understanding (Denham, Renwick-De Bardi, \& Hewes, 1994) and enable the children to discover emotional events, give appropriate responses to emotional stimuli (Eisenberg et al, 1998. Nonsupportive responses of mother(s) include "Minimizing Reactions" (e.g., "don't cry like a baby"), verbal and physical "Punitive Reactions" when children express their emotions verbally (e.g., "Tell my child that If she/he does not stop, otherwise that anything she/he wants to do will not be realized"), and the sixth dimension, 

socialization. International Journal of Human Sciences, 13(1), 2102-2112. doi:10.14687/ijhs.v13i1.3708

"Distress reactions", reflected the degree to which parents respond to the child emotions in a distressing way (e.g., "feel uncomfortable and embarrassed myself"). How parents react against the children's display of emotion, and particularly negative emotions containing responses are of great importance on social emotional functions of children. Punitive or ignoring parental responses affect the children's thoughts over emotions and lead children to regard emotions as more threatening (Eisenberg et al, 1998). In addition, when mothers give more negative and discouraging reactions, emotion understanding levels of preschool children are indicated to be shown less (Denham \& Auerbach, 1995).

As emotion understanding based upon cognitive and social factors is a dynamic and multiple process, it is highly realized with interpersonal interactions in the social environment. Interaction with parents is of great significance in the social and emotional relations of preschool children. Therefore, the parental responses are one of the most substantial indicators affecting the children's emotional development (Dunn, Brown, \& Beardsall, 1991). In the preschool period mothers socializing negative emotions is more complicated compared to socialization of positive emotions for mothers (Ramsden \& Hubbard, 2002). In the light of all these information, in this study, it was aimed to examine the relationship between Turkish children's emotion understanding during preschool period and maternal responses to children's negative emotions. Emotion understanding is also investigated according to children's age and gender.

\section{Method}

\subsection{Research Model}

It can be said that correlational studies are effective in revealing the relations between variables and determining the levels of relationships between variables and they are important studies providing clues for further studies about those relationships (Büyüköztürk, Çakmak, Akgün, Karadeniz \& Demirel, 2009). In order to examine the presence and degree of the two or more variables correlational survey model was conducted in the current study.

\subsection{Study Group}

The participants included 210 Turkish children and their mothers living in Ankara. All children were recruited from kindergartens and they were between 48-72 month-olds. Participants were selected through purposive sampling to obtain maximum variation in information (Patton, 1990). The target children consisted of $105(50 \%)$ girls and $105(50 \%)$ boys. A total of 210 mother's mean age was 35 (ranging from 25 to 48 years). Most of them had university education (\% $53,3)$. The majority had high status occupations $(\% 43,8)$ (i.e. doctor, teacher). The majority of the families $(\% 49,5)$ fell into the medium income category.

\subsection{Data Collection Tools}

Affect Knowledge Test (AKT): The purpose of AKT (Denham, 1986) is to examine the emotional competence of children based upon labeling and recognition of four basic emotions (happy, sad, angry, and afraid) and affective perspective taking based on situational knowledge according to facial expressions with the same four basic emotions. Four felt faces were shown to the children at random. First, children were asked to label a facial expression of each of the four faces (e.g., How does he/she feel ?) in the expressive subtest. Then children were asked to find corresponding to a particular emotion label by pointing (e.g., "Show me the happy face") in the receptive subtest. The affective perspective taking subtest, consists of two types of situations: stereotypical and nonstereotypical. The stereotypical situations include 8 situations (vignette) in 
Kılıç, Ş., \& Güngör Aytar, A. (2016). Emotion understanding of Turkish preschoolers and maternal emotional socialization. International Journal of Human Sciences, 13(1), 2102-2112. doi:10.14687/ijhs.v13i1.3708

which most children normally experience one distinct emotion (e.g., feels happy for icecream). In constrast, nonstereotypical scenarios provide atypical settings in which a puppet character feels opposite emotions to the common individual experience of each child (e.g., feels anger toward a favorite food). Since emotional experiences vary individually, each child was interviewed with different combinations of nonstereotypical situations. Nonstereotypical situations selected based on the results of Parent Questionnaire in the AKT. Two points were given to each correct answer and the incorrect answers got zero points. One point was given to answers which correctly identified the valance of emotion, whether positive or negative (e.g., answering angry instead of sad or nice instead of happy) (Denham et al., 1997).

The Turkish version of AKT was conducted for this study by the researchers. AKT translation and back translation were conducted by five academicians or professionals of developmental psychology, child development and education and early childhood education. Combining together the Turkish and English translations, the common aspects of all were searched and expressions showing difference were expressed in common statements by discussing with translators. The Turkish and English form was given to five professors for examining in terms of content validity, semantic and conceptual equivalence and experiential equivalence. Confirmatory factor analysis was conducted to investigate the construct validity of the Turkish version of the AKT. Each item on the standardized analysis of $t$ values determine whether there is a significant value or not. $t$ values ranged 10.25 and 5.68 and $t$ values for all items were statistically significant $(p<.05)$. Chi-Square value of emotion recognition and emotion labelling subtests $(\chi 2=37.93$, $\mathrm{p}=0.05)$ were significant. The goodness of fit index values of the emotion recognition and emotion labelling subtests was RMSEA $=0.07$, NFI $=0.95, \mathrm{CFI}=0.95, \mathrm{GFI}=0.84$ and AGFI $=0.92$. ChiSquare value of affective perspective taking subtest $\left(\chi^{2}=392, \mathrm{p}=0.05\right)$ was significant. The goodness of fit index values of affective perspective taking subtest (stereotypical and nonstereotypical situations) $\mathrm{RMSEA}=0.08, \mathrm{CFI}=0.93, \mathrm{GFI}=0.84$ and $\mathrm{AGFI}=0.80$. The Cronbach's alpha for emotional labeling and recognition subtests. 64 and for affective perspective taking subtest (stereotypical and non stereotypical situations). 88 was found for the internal consistency.

Coping with Children's Negative Emotions (CCNES): In order to measure maternal socialization of emotion, mothers completed the CCNES. The CCNES contains 12 short hypothetical situations in which children may experience negative emotions such as anger, sadness and fear. Six response categories represent problem-focused, emotion-focused, expressive encouragement, punitive, minimization and distress responses. Parents rate how likely that they would be showing parental responses in each of the five ways. The scale is based on 7-point scale (from very unlikely to likely). The alpha coefficients for the internal consistency of the five subscales were. 78 for problemfocused responses, .80 for emotion- focused responses, .85 for expressive encouragement, .78 for minimization reactions, .69 for punitive reactions, .70 for distress reactions. In the CCNES each of the six mother behaviours represented a conceptually different way of responding to children's negative emotions. Problem-focused responses, emotion-focused responses, expressive encouragement represent supportive maternal responses. Punitive and minimization reactions represent nonsupportive maternal responses (Fabes, Poulin, Eisenberg, Madden-Derdich, 2002). While the first five dimensions of the scale assess parents' specific emotion socialization responses, the sixth one is linked more to the parents' own feeling states rather than to children's feeling states. For this reason, the dimension of Distress Reaction was not examined in the present study. The internal consistency of Cronbach's alpha ranged. 84 for supportive responses and.87 for nonsupportive responses in the Turkish version of the scale (Altan, 2006). 


\subsection{Procedure}

The parent questionnaire, which is a part of AKT was attached the consent form. Mothers firstly filled out this questionnaire which asks them to evaluate typical reactions of their children in spesific situations. Scenario types for the affective perspective taking subtest was selected on the basis of parents' answers to the questionnaire. Before starting the implementation, the necessary legal permissions were received from the Ministry of Education. The aim of the study was explained to the parents and their consent was obtained to involve their children in the research. Each child was tested individually in a quiet room in his/her preschool, kindergarten in suitable days and times. The child was told that if she/he does not want to complete session, he/she has a right to end up the testing. During the sessions if the child seemed inattentive or not responding and talking, the researcher stopped to play or chat with the child for a while. Each child completed AKT tasks in one session and all tasks took approximately 30-40 minute long. After completing the session, the child was thanked, given his or her reward (sticker) and escorted back to his or her classroom.

\subsection{Research Ethics}

In this study, parents did complete a consent form to give permission to the researches. Furthermore, a verbal and official permission asked form the director of preschoolers.

\subsection{Data Analysis}

Confirmatory factor analysis was conducted to investigate the construct validity of AKT. The results of confirmatory factor analysis was submitted in the data tool section. Multivariate two way analysis was performed to explore whether children's emotion understanding performance and mother reported supportive and nonsupportive responses to children's negative emotions differed by children's age and gender. Multivariate one way analysis was conducted to find the differences of emotion understanding and mothers reported responses to children's negative emotions based on mother's education, occupation and family income. Dunnet $C$ and Scheffe Test were used for Post Hoc Comparisons. Pearson correlation coefficients were employed to assess the relations between the children's emotion understanding and the mother's reported responses to children's negative emotions.

\section{Results}

To examine whether maternal responses to children's negative emotions and emotion understanding was related, correlations were conducted between the maternal reported responses and AKT. Unexpectedly, none of the mothers' responses were significantly correlated with children's AKT scores. There was only one and very little significant correlation between emotion labeling subtest and mother's emotion-focused responses $(r=.15, \mathrm{p}<.05)$.

Based on the second aim of this study emotion understanding is also investigated according to children's age and gender. Based on multivariate analysis there was significantly main effect of children's age on emotion recognition, $\left[\mathrm{F}_{(2,204)}=6.870 ; \mathrm{p}<.01\right]$. Effect size of children's age explained $\% 6$ of the total variance of emotion recognition scores. Results of post hoc Dunnett $\mathrm{C}$ analysis reflected that 48-59 month-olds children scores $(\mathrm{M}=7.47, \mathrm{SD}=.793)$, on emotion recognition is less than 60-71 month-olds children scores $(\mathrm{M}=7.81, \mathrm{SD}=.546)$ and 72 and more month-olds children's emotion recognition scores $(\mathrm{M}=7.81, \mathrm{SD}=.519)$. As shown Table 1. multivariate analysis also indicated that there was significantly main effect of children's age on affective perspective taking (stereotypical and nonstereotypical situations), $\left[\mathrm{F}_{(2,204)}=10.162 \mathrm{p}<.01\right]$. 
Kılıç, Ş., \& Güngör Aytar, A. (2016). Emotion understanding of Turkish preschoolers and maternal emotional socialization. International Journal of Human Sciences, 13(1), 2102-2112. doi:10.14687/ijhs.v13i1.3708

Effect size of children's age explained \% 10 of the total variance affective perspective scores. Results of post hoc Dunnett $C$ analysis reflected that 72 and more month-olds scores $(M=38.65$, $\mathrm{SD}=1.80)$ were more than 60-71 month-olds children scores $(\mathrm{M}=37.02, \mathrm{SD}=4.38)$ and 48-59 month-olds children scores $(\mathrm{M}=35.24, \mathrm{SD}=6.11)$. Analysis did not produce significant age differences for emotion labeling scores $\left[\mathrm{F}_{(2,204)}=.615 ; \mathrm{p}>.05\right]$. Multivariate analysis also did not yield and significant gender differences on children's emotion recognition, emotion labeling and affective perspective taking scores.

\begin{tabular}{lllll}
\hline Source & $D f$ & $F$ & $p$ & $\eta$ \\
\hline Emotion labeling & & & & \\
Age & 2 & .615 & .542 & \\
Gender & 1 & .008 & .930 & \\
Age x Gender & 2 & 2.489 & .085 & \\
Error & 204 & & & \\
Total & 210 & & & .06 \\
Emotion Recognition & & & & \\
Age & 2 & 6.870 & $.001^{*}$ & \\
Gender & 1 & 3.461 & .064 & \\
AgexGender & 2 & .080 & .923 & \\
Error & 204 & & & \\
Total & 210 & & & \\
Affective Perspective Taking & & 10.162 & $.000^{*}$ & .10 \\
Age & 2 & .924 & .338 & \\
Gender & 1 & .921 & .400 & \\
AgexGender & 2 & & & \\
Error & 204 & & & \\
Total & 210 & &
\end{tabular}

\section{Discussion}

In the current study, the fact that there are no relations between maternal supportive and nonsupportive responses, and children's emotion understanding can be argued with some findings supporting the result of the present study. There are studies (Song, 2005; Tao, Zhou \& Wang, 2010; Wang, 2003) revealing the different results obtained from Western culture related to the responses of parents to the children's negative emotions. For example, the relation between emotion understanding skills of Korean children in 48-60 month-olds and socialization responses of their mothers to their negative emotions have been examined and it was revealed in that study that maternal supportive and non-supportive responses to their children's negative emotions have no relations (Song, 2005). Wang (2003) also has analyzed the development of emotion situation knowledge of American and Chinese children in 36-72 month-olds as well as the socialization behaviors and practices. Wang (2003) revealed that there are some differences between Chinese and American mothers in terms of socialization behaviors. Tao, Zhou, \& Wang (2010) have reported in study they have performed on Chinese children and their mothers that none of supportive and nonsupportive responses can solely predict the child adjustment variables. These studies bring to mind that "culture" can be an explanatory variable for the current study result as no relations have been detected between the maternal responses and children's emotion understanding. According to Brody \& Hall (2000) socialization is reported to a process dependent to culture and the socialization of child is realized by the members of culture in which the child lives (Vygotsky, 1978, as cited in Mei \& Fernandez, 2004). Gordon (1989) also points out that culture has very substantial effect on the emotion understanding. Nevertheless, Eisenberg et al., (1998) and Thao et al., (2010) 

socialization. International Journal of Human Sciences, 13(1), 2102-2112. doi:10.14687/ijhs.v13i1.3708

have stressed out the fact that parental responses to children's negative emotions, authoritative and democratic parental styles may be altogether evaluated with the developmental mechanisms of socialization (such as, emotional control of children, understanding and expression of emotions). In the current study, contingency, coaching/teaching models have been examined in the basis of "emotion socializing responses" defined as the specific behaviors of mothers for the negative emotions of children (Eisenberg et al, 1998). That no relations have been acquired between the maternal responses and emotion understanding is considered to be more specifically, associated with focus solely on socializing emotions, rather than the qualifications of parents. Socialization beliefs of parents, socialization goals, styles, practices and strategies are all together named as parental characteristics. Furthermore it is reported that parents use these skills in order to contribute to the development of their children (Collins \& Maccoby; Steinberg, Hetherington, \& Bornstein, 2000; Darling \& Steinberg, 1993).

In this study, the fact that no relations were established between maternal supportive and nonsupportive responses and emotion understanding brought to mind that mothers did not try to socialize the negative emotions of their children much. Root \& Rubin (2010) have underlied that mothers tend to make more effort to socialize positive emotions and behaviors. The fact that no relations have been acquired between the maternal supportive responses to children's negative emotions and emotion understanding with nonsupportive responses can be expressed with different reactions simultaneously, as well. For example, if the child is crying, the mother, as a representative of emotion-focused response, can try to calm the child down, and this can be followed by expressive encouragement. Mothers at the same time can exhibit punitive reactions, frequently. Therefore, the mother may deliver one or more reactions which may cause another response (Song, 2005). Gottman et al., (1996) states that emotion-focused responses solely cannot be associated with emotion understanding, and that immediately after emotion-focused responses problem-focused responses should be followed. This situation puts forward the necessity that particularly the combined and subsequent responses of mothers to the negative emotions should include different options for measurements. The fact that no relations have been established in the study between the children's emotion understanding and the responses of mothers can be expressed with the natural development of children's emotion understanding abilities and that the age of the child is of great significance for the emotion understanding. Perez-Rivierea (2008) also emphasized that age is, too, important in the case that no relations have been acquired between families and preschool children for socializing behaviors.

In the last two decades, the studies on emotional and cognitive development have showed that the emotion understanding abilities of children is subject to fundamental developmental changes emerged between 36-72 month-olds (Pons, Harris, \& de Rosnay, 2004). It is also determined in this study that the emotion understanding abilities are increasing depending upon age. This result complies with other studies. For example, Thompson \& Ontai (2002) underline the fact that in the emotion understanding abilities of children, there is a substantial developmental difference from 36 to 60 month-olds. In this increase, not only the age of children but also the other variables are considered to be determiner such as emotional facial expression and verbal clues, accurate assessment of situation based clues, and increase of interactions with other people (Nixon \& Watson, 2001). In this study, children in 72 month-olds have better emotion recognition skills than 48-59 month-olds and 60-71 months-olds. Age based change related to emotion recognition in this study is parallel to the results of other studies. As a result of Denham \& Couchoud (1990) study, performed on children between 24 and 48 month-olds on the emotional facial expressions, it has been concluded that the older children are better compared to younger children. Denham \& Couchoud (1990) also state emotional recognition abilities are increased with age. Widen \& Russell (2004) define that understanding of emotions and recognition processes have developed gradually. Harter \& Buddin (1987) base upon the cognitive development theory of Piaget and point out the 

socialization. International Journal of Human Sciences, 13(1), 2102-2112. doi:10.14687/ijhs.v13i1.3708

fact that when the young children become more developed in cognitive pattern, they can become aware of more than one emotion. The findings of this study have also revealed that 72 month-olds children performed significantly better affective perspective taking skills than younger children. This finding is consistent with many other studies pointing out the fact that the emotional situational knowledge increases with age (Pons \& Harris, 2005). Felleman, Barden, Carlson, Rosenberg \& Masters (1993) stated that facial and voice expressions are very important in the affective perspective taking skills of preschool children. Emotional labeling is not changed in the current study according to the age of the child. Some studies comparing emotion labeling and recognition is complying with the results of this study. In the study of Shields \& Padawer (1983) on 36 and 72 month-olds children, it is stated that labeling emotions is a difficult process. Shields and Padawer (1983) also stated that in order for children to label an emotion, it is necessary to understand the meaning. In addition, in the study of Nelson \& Russell (2008) concerning how 36 and 72 month-olds children label happy, sad, angry and scared facial expressions based upon body and voice clues, it is pointed out that no age based difference and change are available. Additionally, beyond children's age, there is a myriad of other factors such as children's gender that could influence emotion understanding. In this study, it has been revealed that composite scores of AKT of children not changed significantly according to gender. Martin \& Green (2005) indicated that emotion understanding skill is not different according to gender. It should be researched why there are no gender based differences in children's understanding of emotions in different cultures according to Lenti-Boero \& Giacobbe (1999) and Perez-Riviera (2008).

\section{Conclusion and Suggestions}

For future studies, it may be useful to include other important predictors of children's social and emotional competence. First, paternal responses can be studied as well as maternal responses in other studies. Second, those studies to be realized for the parental socialization can focus on parental variables such as stress levels of parents, marital relations, social and interpersonal skills. Third, it is important to obtain data concerning the social emotional skills from independent resources (mother, child and teacher) with independent measures. In the current study maternal responses to children's negative emotions measured based on their self report. Obtaining data with many methods (e.g., standard test, questionnaire, semi-structured observation etc.) will contribute to the multiple dimensional relations. Based upon the supportive and nonsupportive response reports, and observations conducted at home, laboratory or school environment, substantial contribution will be enabled. Fifth, it is important to note that other components of emotion understanding should be added in future researches. In this study emotion understanding skills have been examined upon emotion recognition, labeling and affective perspective taking. Future studies can include evaluations from many other aspects such as external causes, memory and belief.

\section{Limitation of the study}

The current study was conducted with 48-72 month-olds and their mothers in Ankara city center. Therefore it can be explain the limitation of the study was the age group of preschoolers and their mothers. 
Kılıç, Ş., \& Güngör Aytar, A. (2016). Emotion understanding of Turkish preschoolers and maternal emotional socialization. International Journal of Human Sciences, 13(1), 2102-2112. doi:10.14687/ijhs.v13i1.3708

\section{References}

Altan, Ö. (2006). The Effects of Maternal Socialization and Temperament on Children's Emotion Regulation. Unpublished master thesis, Koç University, İstanbul.

Brody, L. R., \& Hall, J. (2000). Gender, emotion, and expression. In M.Lewis and J. Haviland-Jones (Eds.), Handbook of Emotions (2nd ed., pp. 338-349).New York: Guilford.

Bronson, M. B. (2000). Self-regulation in early childhood. Nature and nurture. NewYork: Guilford.

Büyüköztürk, S., Kılıç Çakmak, E., Akgün, Ö. E., Karadeniz, S.., \& Demirel, F. (2008). Bilimsel Araştırma Yöntemleri [Academic Research Methods], Ankara: Pegem Akademi

Camras, L. A., \& Allison, K. (1985). Children's understanding of emotional facial expressions and verbal labels. Journal of Nonverbal Behavior, 9, 84-94.

Collins, W. A., McCoby, E. E.,Steinberg, L., Hetherington, E. M., \& Bornstein, M. H. (2000). Contemporary research on parenting: The case for nature and nurture. American Pscychologist, $55,218-232$.

Cutting, A. L., \& Dunn, J. (1999). Theory of mind, emotion understanding, language and family background: Individual differences and interrelation. Child Development, 70, 853-865.

Darling, N., \& Steinberg, L. (1993). Parenting style as context: An Integrative Model. Psychological Bulletin,113,487-496.

Denham, S. A. (1986). Social cognition, prosocial behavior and emotions in preschoolers: Contextual validation. Child Development, 57, 194-201.

Denham, S. A. (1998). Emotional Development in Young Children. The Guilford Series on Social and Emotional Development. New York: Guilford.

Denham, S., \& Auerbach, S. (1995). Mother-child dialogue about emotions. Genetic, Social, and General Psychology Monographs, 121, 301-319.

Denham, S. A., Bassett, H. H., \& Wyatt, T. M. (2010). Gender differences in the Socialization of preschoolers' emotional competence. New Directions for child and adolescent development, 128, $29-49$.

Denham, S. A., Blair, K. A, DeMulder, E.Levitas, J., Sawyer, K., \& Auerbech-Major, S. V. (2003). Preschool emotional competence: Pathway to social competence?. Child Development, 74, 238-256.

Denham, S. A., \& Couchoud, E. A. (1990). Young preschoolers' understanding of emotion. Child Study Journal, 20, 171-192.

Denham, S. A., \& Grout, L. (1993). Socialization of emotion: Pathway to preschoolers' emotional and social competence. Journal of Nonverbal Behavior,17, 205-227.

Denham, S. A., Mitchell-Copeland, J., Strandberg, K. Auerbach, K. S., \& Blair, K. (1997). Parental contributions to preschoolers' emotional competence: Direct and indirect effects. Motivation and emotion, 21, 65-86.

Denham, S. A., Renwick-DeBardi, S., \& Hewes, S. (1994). Emotional communication between mothers and preschoolers: Relations with emotional competence. Merrill Palmer Quarterly, 40, 488-508.

Dunn, J., Bretherton, I., \& Munn, P. (1987). Conversations about feeling states between mothers and their young children. Developmental Psychology, 23, 132-139.

Dunn, J., Brown, J, \& Beardsall, L. (1991). Family talk about feelings states and children's later understanding of other's emotions. Developmental Pschology, 27, 3, 448-455.

Dunn, J., \& Hughes, C. (1998). Young children's understanding of emotions within close relationships. Cognition and Emotion, 12, 171-190.

Eisenberg, N., Cumberland, A., \& Spinrad, T. L. (1998). Parental socialization of emotion. Psychological Inquiry, 9, 241-273.

Eisenberg, N., Fabes, R. A., \& Murphy, B. C. (1996). Parent's reactions to children's negative emotions: Relations to children's social competence and comforting behavior. Child Development, 67, 2227-2247. 
Kılıç, Ş., \& Güngör Aytar, A. (2016). Emotion understanding of Turkish preschoolers and maternal emotional socialization. International Journal of Human Sciences, 13(1), 2102-2112. doi:10.14687/ijhs.v13i1.3708

Fabes, R. A., Leonard, S. A., Kupanoff, K., \& Martin, C. L. (2001). Parental coping with children's negative emotions: Relations with children's emotional and social responding. Child Development, 72(3), 907-920.

Fabes, R. A., Poulin, R., Eisenberg, N., \& Madden-Derdich, D. A. (2002). The coping with children's negative emotions Scale (CCNES): Psychometric Properties and Relations with Children's emotional competence. Marriage and Family Review, 34, 285-310.

Felleman, E. S., Barden, R. C., Carlson, C. R., Rosenberg, L., \& Masters, J. C. (1993).Children's and adults' recognition of spontaneous and posed emotional expressions in young children. Developmental Psychology, 19, 405-413.

Gnepp, J. (1983). Children's social sensitivity: Inferring emotions from conflicting cues. Developmental Psychology, 19, 805-814.

Gordon, S. L. (1989). The socialization of children's emotions: Emotion culture, competence, and exposure. In C. Saarni and P. L.Harris (Eds.), Children's Understanding of Emotions. Cambridge, England: Cambridge University.

Gottman, J. M., Katz, L. F., \& Hooven, C. (1996). Parental meta-emotion philosophy and the emotional life of families: Theoretical models and preliminary data. Journal of Family Psychology, 10 (3), 243-268.

Gomez-Guerrero, S. (2003). Emotional understanding in clinical and normal children in correlation with mother's emotional awareness and attitudes toward children expressiveness. Unpublished Doctoral Dissertation, Universite de Montreal Faculte des etudes superieuires, Canada.

Harris, P. L. (1989). Understanding emotion and experiencing emotion. In C. Saarni (Ed.), Children's understanding of emotion (pp. 241-258). Cambridge: Cambridge University Press.

Harter, S., \& Buddin, B. J. (1987). Children's understanding of simultaneity of two emotions: a five stage developmental acquisition sequence. Developmental Psychology, 23, 3, 388-399.

Lenti,C., Lenti-Boero,D., \& Giacobbe, A. (1999). Decoding of emotional expression in children and adolescents. Perceptual and Motor Skills,89,804-814.

Martin, R. M., \& Green, J. A. (2005). The use of Emotion explanations by mothers: Relation to preschoolers's gender and understanding of emotions. Social Development, 14-2.

Mei, G., \& Fernandez, C. (2004). Talking about past emotions: Conversations between Peruvian Mothers and their preschool children. Sex Roles, 50, (9/10), 641-657.

Meerum, T, M., \& Olthof, T. (1989). Awareness and self-regulation of emotion in young children. In C.Saarni, \& P. Harris (Eds.). Children's Understanding of emotion. New York: Cambridge University.

Nelson, N, L., \& Russell, J. A. (2008). Children's labeling of dynamic facial, postural and vocal cues to emotion. Presented at the Annual Meeting of the Association for Psychological Science.

Nixon, C. L., \& Watson, A. C. (2001). Family experiences and early emotion understanding. MerrillPalmer Quartely, 47, 300-322.

Patton, M. (1990). Qualitative evaluation and research methods. (pp. 169-186). Beverly Hills, CA: Sage. Designing Qualitative Studies.

Perez-Rivera, M. B. (2008). Mother's belief about emotions, mother-child emotion discourse and children's emotion understanding in Latino Families. Unpublished master's thesis. Blacksburg, VA.

Pons, F., \& Harris, P. L. (2005). Longitidunal change and longitudinal stability of individual diffrences in children's emotion understanding. Cognition and Emotion,19 (8),1158-1174.

Pons, F., Harris, P. L., \& de Rosnay, M. (2004). Emotion comprehension between 3 and 11 years: Developmental periods and hierarchical organization. European Journal of Developmental Psychology, 2004, 1(2), 127-152.

Ramsden, S. R., \& Hubbard, J. A. (2002). Family expressiveness and parental emotion coaching: Their role in children's emotion regulation and aggression. Journal of Abnormal Child Psychology, 30, 657-667.

Root, A. K., \& Rubin, K. H. (2010).Gender and Parent reactions to children's emotion during preschool years. In A. Kennedy Rootand S. Denham (Eds.), The role of gender in the 
Kılıç, Ş., \& Güngör Aytar, A. (2016). Emotion understanding of Turkish preschoolers and maternal emotional socialization. International Journal of Human Sciences, 13(1), 2102-2112. doi:10.14687/ijhs.v13i1.3708

socialization of emotion: Key concepts and critical issues. New Directions for Child and Adolescent Development,128,51-64. San Francisco:Jossey-Bass.

Shields, S. A., \& Padawer, J. A. (1983). Children's standards for judging their own facial expressions of emotion. Journal of Nonverbal Behavior, 8, 109-125.

Smith, M., \& Walden, T. (1998). Developmental trends in emotional understanding among a diverse sample of African-American preschooler children. Journal of Applied Developmental Psychology,19, 177-197.

Song, H. (2005). The relationships between parental reactions to children's negative emotions and children emotion understanding: mediated pathways by children internal working models od attachment relationship. Unpublished master's thesis. University of Wisconsin, Madison.

Tao, A., Zhou, Q., \& Wang, Y. (2010). Parental reactions to children's negative emotions. Journal of Family Psychology, 24, 2, 135-144.

Thompson, R. A., \& Ontai, L. L. (2002). Patterns of attachment and maternal discourse effects on children's emotion understanding from 3 to 5 years of age. Social Development, 11, 4, 433450.

Wang, Q. (2003). Emotion situation knowledge in American and Chinese preschool children and adults. Cognition and Emotion, 17, 5, 725-746.

Widen, S. C., \& Russell, J. A. (2004). The relative power of on emotion's facial expression, label, and behavioral consequences to evoke preschoolers' knowledge of its cause. Cognitive Development, 19, 111-125. 